\title{
Spectroscopic and photometric confirmation of chromospheric activity in four stars
}

\author{
O. Özdarcan ${ }^{1, \star}$ and H. A. Dal ${ }^{1}$ \\ Ege University, Science Faculty, Department of Astronomy and Space Sciences, 35100 Bornova, İzmir, Turkey. \\ Received XXXX, accepted XXXX \\ Published online XXXX
}

Key words stars: activity - stars: fundamental parameters - stars: individual (BD+13 5000, BD+11 3024, TYC 3557919-1, TYC 5163-1764-1) - stars: late-type

We present analysis of medium resolution optical spectra and long term $V$ band photometry of four cool stars, BD+13 5000, BD+11 3024, TYC 3557-919-1 and TYC 5163-1764-1. Our spectroscopic analysis reveals that the stars are giant or sub-giant from K0 or K1 spectral type, and all of them exhibit emission features in their Ca II H\& K lines. These features appear to be modulated with the rotation of the stars. Except BD+11 3024, we observe that the radial velocities of the target stars are not stable, which suggests that each of them might be a member of a binary system. Global analysis of photometric data indicates clear cyclic variation for BD+13 5000 and TYC 5163-1764-1 with a period of 8.0 0.3 and $5.04 \pm 0.04$ year, respectively. Besides that, we observe a dramatic increase $(\sim 0.7)$ in the mean brightness of BD +113024 , accompanied with a $2.87 \pm 0.12$ cyclic variation, embedded into the global brightening trend, which indicates possible multiple cycles on this star.

Copyright line will be provided by the publisher

\section{Introduction}

Advent of the Automatic Photoelectric Telescopes (APT, see, e.g. Henry \& Eaton 1995; Strassmeier et al. 1997) enabled precise and efficient long term photometric observations of chromospherically active stars, which exhibit emission in their $\mathrm{Ca}$ II $\mathrm{H} \& \mathrm{~K}$ lines. Observed stars are usually brighter than 10 magnitude in $V$ band. Nowadays, these stars have continuous photometry for more than twenty years, which were used in many studies on analysing photometric activity cycles (Jetsu et al.2017, Oláh et al.2009), indirect surface imaging (Roettenbacher et al.2011), and relation between light curve properties and photometric periods (see, e.g. Fekel \& Henry 2005; Fekel et al. 2002; Özdarcan et al. 2010).

Further contribution in terms of collecting continuous photometry came from photometric surveys, such as The All Sky Automated Survey (ASAS, Pojmanski 1997, 2002, Pojmanski et al.2005), and Northern Sky Variability Survey (NSVS, Woźniak et al. 2004). From these databases, numerous variable stars, which are fainter relative to the stars observed by APTs, were discovered. BD+11 3024, BD+13 5000, TYC 3557-919-1 and TYC 5163-1764-1 are such targets.

BD+11 3024 exhibits strong X-ray emission (Zickgraf et al. 2003) and photometric variability with a period of 21.69 (Bernhard 2008). TYC 3557-919-1 was listed in the catalogue of Haakonsen \& Rutledge (2009), where the nearinfrared counter parts of X-ray sources in ROSAT Bright

^ Corresponding author: orkun.ozdarcan@ege.edu.tr
Source Catalogue (Voges et al. 1999) were presented. Photometric variability of the star was reported by Bernhard (2008) with a period of $25^{\mathrm{d}} .08$. BD+13 5000 took place in the catalogue published by Boyle et al. (1997), which provides optical counterpart of some X-ray sources in the ROSAT catalogue. Hoffman et al. (2009) identified the star as a new variable. Kiraga (2012) identified it as a rotating variable with a period of 18.14 , while Lloyd et al. (2011) classified the star as chromospherically active. Among our target stars in this work, TYC 5163-1764-1 has the least literature information. The star was identified as an X-ray source in ROSAT catalogue (Voges et al. 1999), and then its photometric variability was reported by Lloyd et al. (2011) with a variation period of 26.08 . All the four targets have no detailed study on their atmospheric properties and photometric characteristics.

In the scope of this study, we obtained medium resolution optical spectra of these targets to determine their spectral characteristics and investigate spectroscopic binarity of them. Furthermore, we collected differential $V$ band photometry of the targets from three different sources. Collected data enabled us to investigate seasonal and long term photometric behaviour of the targets, as well as their seasonal photometric periods. In the next section, we describe our spectroscopic observations, data reductions and analysis. Sources of collected photometric data, together with global and seasonal photometric analysis are given in Section 3 . In the last section, we summarize our findings on each target. 


\section{Spectroscopy}

\subsection{Observations and data reduction}

We carried out optical spectroscopic observations of the program stars with Turkish Faint Object Spectrograph Camera (TFOSC ${ }^{1}$ ) attached to the $1.5 \mathrm{~m}$ Russian - Turkish telescope at TÜBİTAK National Observatory (TNO). Back illuminated $2048 \times 2048$ pixels CCD camera with a pixel size of $15 \times 15 \mu^{2}$ was used together with the spectrograph. Using échelle mode of TFOSC, we were able to record spectra between $3900-9100 \AA$ in 11 échelle orders, which provided spectral resolution of $\mathrm{R}=\lambda / \Delta \lambda \sim 2800$ around 6500 Å.

We follow standard procedure for reducing échelle spectra, which basically includes bias correction, flat-field division of Fe-Ar calibration frames and science frames, followed by scattered light correction and cosmic rays removal, and finally extraction of spectra from échelle orders. Wavelength calibration of reduced and extracted science spectra are done via Fe-Ar images, and wavelength calibrated science spectra are normalized to the unity by using 4th or 5th order cubic spline function.

\subsection{Radial velocities}

We give a brief log of spectroscopic observations in Table 1 . Note that, in addition to the target star observations, we obtained optical spectra of 35 Peg (K0 III) and $\theta$ Psc (K1 III) with the same instrumental set-up, and used them as spectroscopic comparison and radial velocity template. We measure radial velocities of the targets by cross-correlating each of their spectrum with the observed templates using the technique described in Tonry \& Davis (1979). We apply the technique by using fxcor task (Fitzpatrick 1993) of IRAH ${ }^{2}$ software. We use $\theta$ Psc as radial velocity template for $\mathrm{BD}+135000$, while 35 Peg is the template for the remaining target stars. Resulting cross-correlation functions exhibit sharp and single peak for each target, indicating either these stars are single, or SB1 systems which can not be resolved in our resolution.

Inspecting Table 1, one can notice non-stable radial velocities for each target. Difference between measured maximum and minimum radial velocities is the smallest and about $17 \mathrm{~km} \mathrm{~s}^{-1}$ for $\mathrm{BD}+113024$, but much larger for the others. This could be result of a motion on a Kepler orbit, i.e. each star may actually be a member of an SB1 system. Although the clear difference between measured radial velocities of each individual star, we can not check this possibility with a preliminary orbital solution due to the less number of observed spectra sparsely distributed in time. However, we plot phase-folded radial velocities of each target in the appendix, for visual evaluation of radial velocity patterns

\footnotetext{
1 http://www.tug.tubitak.gov.tr/rtt150_tfosc.php

2 The Image Reduction and Analysis Facility is hosted by the National Optical Astronomy Observatories in Tucson, Arizona at URL iraf.noao.edu.
}

Table 1 Log of spectroscopic observations. Exposure time is in second, signal-to-noise ratio $(\mathrm{S} / \mathrm{N})$ is measured around $5500 \AA$. Radial velocity $\left(\mathrm{V}_{r}\right)$ and its standard error $\left(\sigma_{V_{r}}\right)$ are given in $\mathrm{km} \mathrm{s}^{-1}$.

\begin{tabular}{cccccc}
\hline Star & $\begin{array}{c}\text { HJD } \\
(2457000+)\end{array}$ & $\begin{array}{c}\text { Exp. } \\
(\mathrm{s})\end{array}$ & $\mathrm{S} / \mathrm{N}$ & $\mathrm{V}_{r}$ & $\sigma_{V_{r}}$ \\
\hline & 378.2194 & 3600 & 208 & -39.3 & 4.1 \\
& 604.5612 & 3600 & 138 & -43.9 & 2.4 \\
BD+135000 & 605.4999 & 3600 & 177 & -37.3 & 5.8 \\
& 671.4607 & 3600 & 239 & 32.9 & 2.9 \\
& 672.4661 & 3600 & 225 & 46.3 & 1.6 \\
\hline & 604.4006 & 3600 & 107 & -38.8 & 2.0 \\
& 605.4410 & 3600 & 122 & -36.1 & 2.2 \\
TYC 3557-919-1 & 671.4051 & 3600 & 182 & 9.8 & 1.7 \\
& 672.4114 & 3600 & 166 & 12.9 & 2.2 \\
& 853.5443 & 1095 & 98 & -24.4 & 2.2 \\
TYC 5163-1764-1 & 854.4724 & 3200 & 70 & -27.3 & 3.4 \\
\hline & 604.4871 & 3600 & 107 & 36.1 & 4.2 \\
& 605.3279 & 3600 & 41 & 27.4 & 4.3 \\
& 671.3019 & 3600 & 165 & -13.2 & 3.3 \\
& 672.2491 & 3600 & 179 & -12.2 & 3.5 \\
\hline BD+113024 & 604.3434 & 3600 & 200 & 11.1 & 3.9 \\
& 605.3820 & 3600 & 200 & 8.9 & 4.6 \\
& 832.6153 & 3600 & 248 & 3.9 & 2.7 \\
& 854.4324 & 2700 & 276 & -6.3 & 4.3 \\
\hline
\end{tabular}

with respect to the calculated photometric periods (see Section 3.3.2 and Table 5p.

\subsection{Spectral types and features}

We compare spectra of target stars with the observed template spectra of 35 Peg and $\theta$ Psc to determine spectral type of each target. We choose a single observed spectrum for each target, which has the highest $\mathrm{S} / \mathrm{N}$, for comparison. In the first step we observe that the spectrum of $35 \mathrm{Peg}$ provides close match to the spectra of TYC 3557-919-1, TYC 5163-1764-1 and BD+11 3024, thus indicating K0 III spectral type, while spectrum of $\theta$ Psc is similar to the spectra of BD+135000 and pointing out K1 III spectral type. In the second step, we adopt previously determined atmospheric parameters of 35 Peg and $\theta$ Psc (Sharma et al.2016), as initial atmospheric parameters of corresponding target star, and switch to the spectrum synthesizing method to find the best matching synthetic spectrum to the observed spectrum of each target. Atmospheric parameters of the best matching synthetic spectrum is adopted as the final atmospheric parameters of the corresponding target.

We use the latest version of python framework iS pec (Blanco-Cuaresma et al. 2014), which includes different spectrum synthesis codes, model atmospheres and line lists. In our case, we adopt SPECTRUM ${ }^{3}$ code (Gray \& Corbally 1994) in conjunction with MARCS model atmospheres (Gustafsson et al. 2008) and the updated line list from Vienna Atomic Line Database (VALD, Ryabchikova et al. 2015). We calculate synthetic spectra for effective temperatures between $4000-5500 \mathrm{~K}$ with a step of $250 \mathrm{~K}, \log g$ value between $2.0-4.5$ with a step of 0.5 , and metallicity

\footnotetext{
${ }^{3}$ http://www.appstate.edu/ grayro/spectrum/spectrum.html
} 

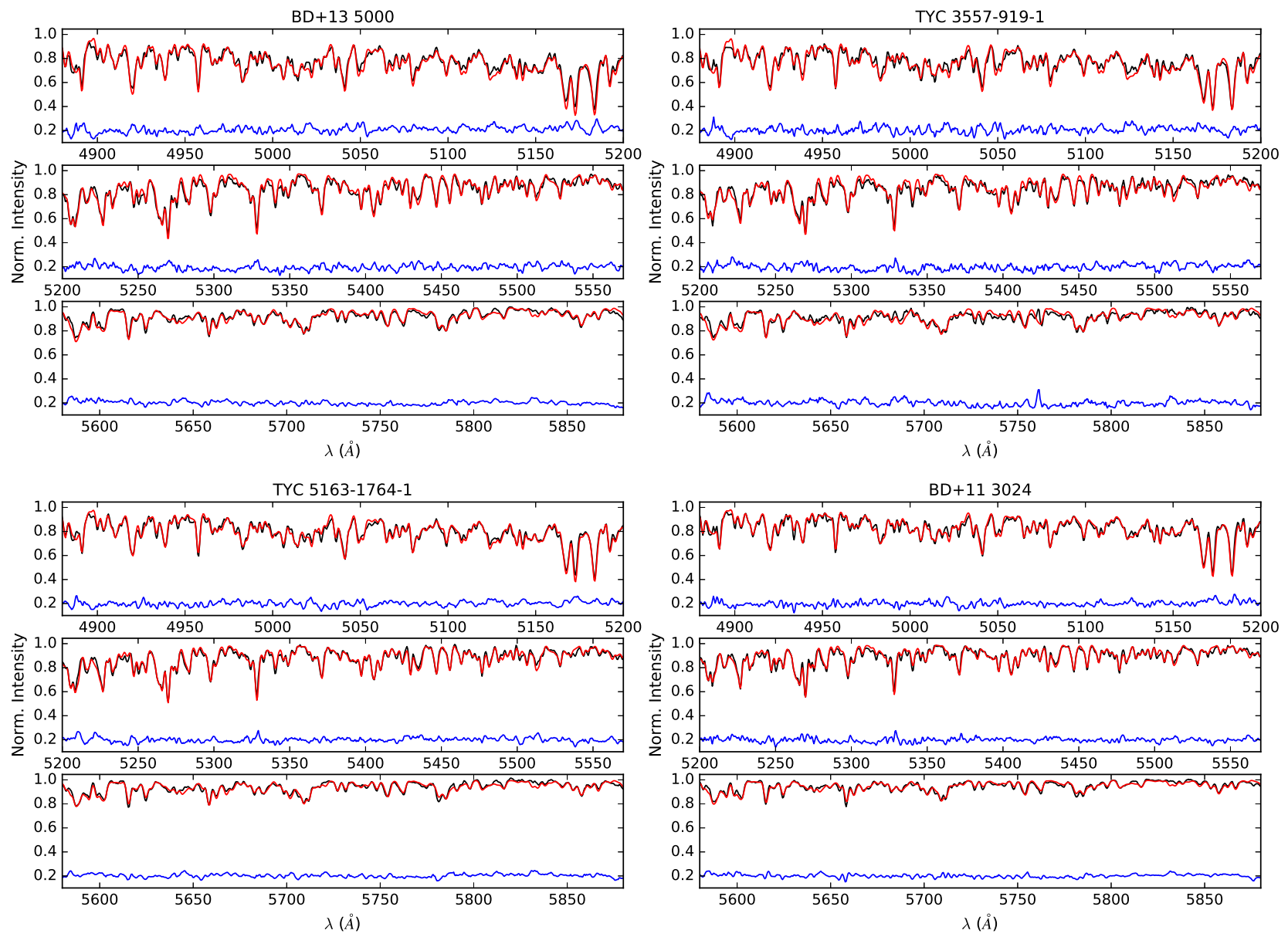

Fig. 1 Observed (black) and best-matched synthetic spectrum (red) for each target stars. Residuals from the best-matched spectrum are shown in blue and shifted by 0.2 upwards for the sake of simplicity.

values $([\mathrm{M} / \mathrm{H}])$ between -1.0 and 0.0 in steps of 0.25 . We convolve each synthesized spectrum with a proper Gaussian line-spread function to match the resolution of TFOSC spectra. Then, we re-normalize the observed spectrum with respect to the instrumentally broadened synthetic spectrum, in order to compensate continuum level uncertainty coming from normalization by cubic splines in IRAF. Finally, we compare synthesized spectrum with the re-normalized observed spectrum by considering residuals. Since numerical fitting methods are not very efficient due to the resolution of TFOSC spectra, we only calculate synthetic spectra for available MARCS models, and refrain from any interpolation between the available model atmospheres. In the whole process, we keep micro-turbulence velocity fixed at 2 $\mathrm{km} \mathrm{s}^{-1}$. Considering resolution and $\mathrm{S} / \mathrm{N}$ of TFOSC spectra, and grid steps of MARCS models for effective temperature, $\log g$ and metallicity, we estimated uncertainties as $200 \mathrm{~K}$ in $T_{\text {eff }}, 0.5$ in $\log g$ and 0.25 in $[\mathrm{M} / \mathrm{H}]$. We list the atmospheric parameters of the synthetic spectra, that provides the closest match to the observed spectra of the target stars, in Table 2 In Figure 11, we plot observed and best-matched spectra at optical wavelengths, together with residuals from the bestmatched synthetic spectra.
Table 2 Final atmospheric parameters of target stars. Sp denotes estimated spectral type.

\begin{tabular}{lcccl}
\hline Star & $\begin{array}{c}T_{\text {eff }} \\
(\mathrm{K})\end{array}$ & $\begin{array}{c}\log g \\
(\mathrm{cgs})\end{array}$ & {$[\mathrm{M} / \mathrm{H}]$} & Sp. \\
\hline BD+13 5000 & 4750 & 3.5 & 0.0 & K0IV \\
TYC 3557-919-1 & 4750 & 3.0 & 0.0 & K0III-IV \\
TYC 5163-1764-1 & 4500 & 2.5 & -0.5 & K2III \\
BD+11 3024 & 4750 & 3.0 & -0.5 & K0III-IV \\
\hline
\end{tabular}

We observe noticeable spectral characteristics of $\mathrm{H}_{\alpha}$ and $\mathrm{Ca}$ II $\mathrm{H} \& \mathrm{~K}$ lines, which are sensitive to the chromospheric activity in cool stars. In Figure 2, we plot observed spectra around these lines. For all stars, Ca II H\& K lines are in form of emission. Very strong emission in Ca II $\mathrm{H} \& \mathrm{~K}$ lines, which exceeds continuum, and varying $\mathrm{H}_{\alpha}$ profiles are remarkable for BD+135000 and TYC 3557-919-1, while $\mathrm{BD}+113024$ and TYC 5163-1764-1 show variable $\mathrm{H}_{\alpha}$ line profiles, but usually in form of filled absorption, which can be concluded by comparing their line profiles with the template star spectrum. Considering variable strength of emission features at different observation epochs, these features 
appear to be modulated with the rotation of the star. These spectral features and atmospheric parameters of the target stars clearly indicate chromospheric activity.

\section{Photometry}

We collect differential $V$ photometry of our target stars with respect to a proper nearby comparison and check stars. We list basic data of all program stars in Table 3

Differential $V$ photometry comes from three sources. The first and the second sources are The All Sky Automated Survey (ASAS), and All-Sky Automated Survey for Supernovae Sky Patrol (ASAS-SN, Kochanek et al. 2017, Shappee et al. 2014) databases, and the third source is our Bessell $V$ CCD observations obtained at TNO.

\subsection{ASAS and ASAS-SN photometry}

We extract all available $V$-band photometric data of program stars from ASAS and ASAS-SN databases. Since we only have differential magnitudes from T60 observations, we convert standard magnitudes to differential magnitudes in order to evaluate observations on a common scale. We calculate average standard $V$ magnitude of each comparison star in the databases, and subtract it from each individual standard $V$ magnitudes of corresponding target star, thus obtain differential magnitudes in the sense of variable-minuscomparison. We estimate the precision of differential magnitudes with respect to the check-minus-comparison magnitudes. For check-minus-comparison measurements, we consider observing nights where simultaneous measurements from both check and comparison stars are available at exactly the same Julian date, and then obtain check-minuscomparison magnitudes. We list a brief information on collected data from ASAS and ASAS-SN databases in Table 4

\subsection{T60 photometry}

We collect Bessell $V$ CCD observations with $0.6 \mathrm{~m}$ robotic telescope (T60) at TNO, equipped with $2048 \times 2048$ pixels FLI ProLine 3041-UV CCD camer ${ }^{4}$ with a pixel size of $15 \times 15 \mu^{2}$. We reduce automatically obtained CCD images with $c c d r e d$ package in IRAF environment, following bias subtraction and then flat field division of science frames. The CCD has an efficient cooling system, which cools down the CCD to $-60^{\circ} \mathrm{C}$ and strictly stabilizes it during the whole night, hence bias and dark frame counts are almost same and we do not apply dark subtraction. We extract magnitudes of program stars from reduced science frames with phot package in IRAF. Since comparison stars are just a few arc minutes away from target stars, we did not apply atmospheric extinction correction. In Table 4, we tabulate the brief information on our T60 observations.

\footnotetext{
4 http://www.tug.tubitak.gov.tr/t60_fli_ccd.php
}

We present T60, ASAS and ASAS-SN $V$ observations in Figure 3 Lower precision of ASAS measurements is noticeable compared to the T60 and ASAS-SN observations. Although the observational scatter can be as large as amplitude of individual light curves for ASAS data, it still confirms global stability of comparison stars, but with lower precision. T60 and ASAS-SN data provide further confirmation for the stability of comparison stars, with better precision.

\subsection{Photometric analysis}

\subsubsection{Global photometric variation}

We observe variable mean brightness and light curve amplitude for all targets. BD+13 5000 and TYC 5163-1764-1 exhibit cyclic mean brightness variation in time. Application of Lomb-Scargle periodogram (Lomb 1976; Scargle 1982) using scipy package under Python environment leads to $8.0 \pm 0.3$ and $5.04 \pm 0.04$ year cycle period for $\mathrm{BD}+135000$ and TYC 5163-1764-1, respectively. No evidence is found for mean brightness variation with a longer period. In the case of TYC 3557-919-1, collected data cover only four years of time range, and variation pattern does not show any cyclic variation, but a $\sim 0^{\mathrm{m}} 1$ global brightening trend.

On the other hand, BD+113024 exhibits a striking increase in global brightness in ASAS photometry. The amount of increase is $\sim 0.7$ and this increase is accompanied with a variable light curve amplitude from season to season. T60 and ASAS-SN observations show that the star somehow maintains its maximum brightness observed at the end of ASAS data. We apply second order polynomial fit to the ASAS data of the star, and linear fit to ASAS-SN and T60 data, to de-trend the whole light curve, and search for a signal of any cyclic brightness variation. Application of Lomb-Scargle periodogram to the de-trended data indicates $2.87 \pm 0.12$ year cyclic variation with an amplitude of 0.03 , which is shown in Figure 4

\subsubsection{Photometric period and seasonal brightness variations}

For each target, we determine minimum, maximum and mean brightnesses of seasonal light curves, as well as corresponding light curve amplitudes and photometric periods. In most cases, light curves are asymmetric, which is a common feature observed in light curves of chromospherically active stars. Hence, application of periodogram methods based on Fourier transform would result in multiple periods, i.e. main period, which corresponds to the photometric period, and its harmonics. However, application of statistical methods, such as Phase Dispersion Minimization (PDM, Stellingwerf 1978) and Analysis of Variances (ANOVA, Schwarzenberg-Czerny 1996), are more efficient to find a single period which would be the single representative period for the observed variation, without any additional periods (i.e. harmonics). In the scope of our study, we adopt 

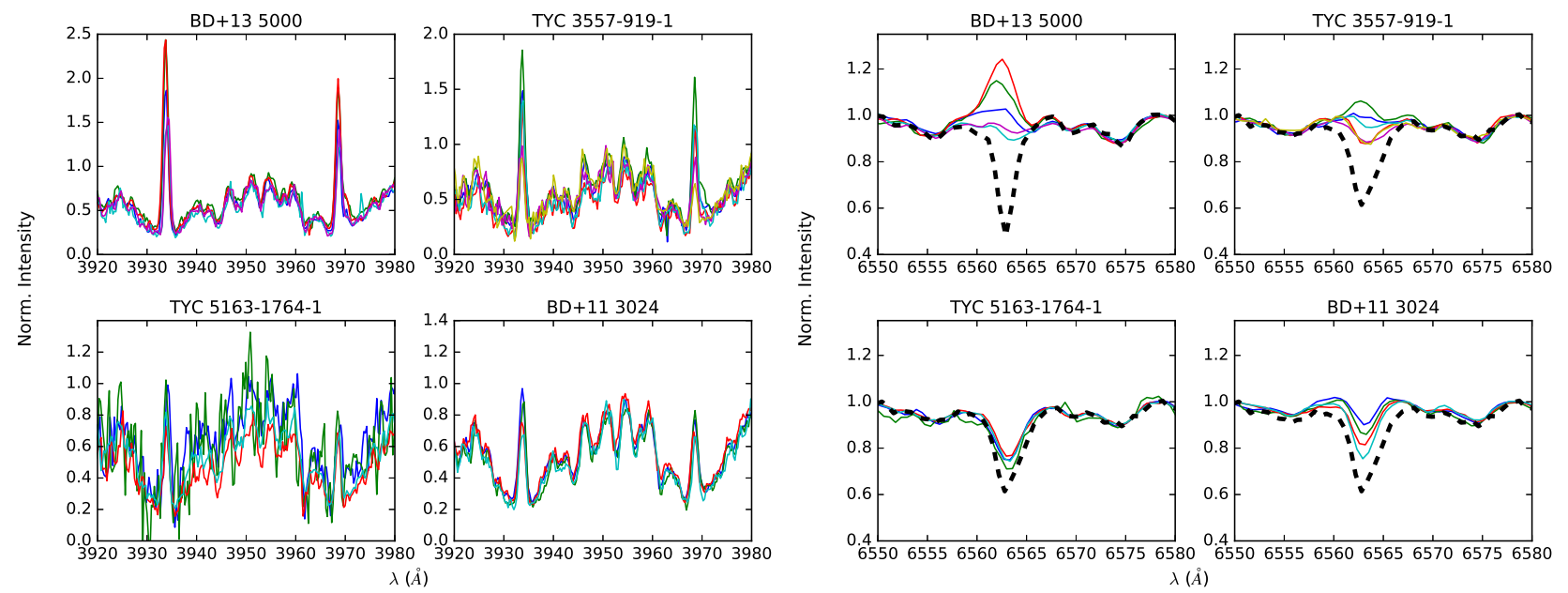

Fig. 2 Observed spectra around $\mathrm{Ca}$ II $\mathrm{H} \& \mathrm{~K}$ lines (left four plots) and $\mathrm{H}_{\alpha}$ line (right four plots). For each star, we plot all observed spectra with continuous lines in different colors, indicating different Julian dates given in Table 1 . In $\mathrm{H}_{\alpha}$ plots, note that we over plot observed spectrum of 35 Peg with thick dashed line for BD+13 5000, while the same thick dashed line denotes observed spectrum of $\theta$ Psc in the remaining plots.

Table 3 Basic data of program stars. $V$ magnitudes and $B-V$ colours are from Tycho-2 catalogue (Høg et al. 2000), while $J H K$ magnitudes are from 2MASS All Sky Catalog of point sources (Cutri et al. 2003).

\begin{tabular}{|c|c|c|c|c|c|c|c|c|}
\hline Star & Identifier & $\alpha(2000)$ & $\delta(2000)$ & $V$ & $B-V$ & $J$ & $H$ & $K$ \\
\hline Variable & $\mathrm{BD}+135000$ & $22^{h} 50^{m} 24^{s}$ & $+14^{\circ} 31^{\prime} 43^{\prime \prime}$ & 10.61 & $1 . \mathrm{m} 13$ & 8.455 & 7.895 & $7 \cdot .748$ \\
\hline Comparison & 2MASS $22502241+1434264$ & $22^{h} 50^{m} 22^{s}$ & $+14^{\circ} 34^{\prime} 26^{\prime \prime}$ & - & - & $11^{\mathrm{m}} \cdot 105$ & $10^{\mathrm{m}} 946$ & 10.858 \\
\hline Check & $\mathrm{BD}+135001$ & $22^{h} 50^{m} 30^{s}$ & $+14^{\circ} 36^{\prime} 45^{\prime \prime}$ & 9.94 & 0.31 & 9.276 & $9^{\mathrm{m}} 183$ & 9.124 \\
\hline Variable & TYC 3557-919-1 & $19^{h} 43^{m} 40^{s}$ & $+46^{\circ} 40^{\prime} 03^{\prime \prime}$ & 11.22 & 1.00 & 9.232 & 8.684 & 8. 536 \\
\hline Comparison & 2MASS $19432561+4641206$ & $19^{h} 43^{m} 26^{s}$ & $+46^{\circ} 41^{\prime} 21^{\prime \prime}$ & - & - & $10^{\mathrm{m}} .135$ & 9.730 & 9.617 \\
\hline Check & 2MASS $19430979+4642507$ & $19^{h} 43^{m} 10^{s}$ & $+46^{\circ} 42^{\prime} 51^{\prime \prime}$ & - & - & 11.947 & 11.852 & 11.827 \\
\hline Variable & TYC 5163-1764-1 & $20^{h} 23^{m} 34^{s}$ & $-01^{\circ} 45^{\prime} 02^{\prime \prime}$ & 11.335 & 1.33 & - & - & - \\
\hline Comparison & TYC 5163-1960-1 & $20^{h} 23^{m} 06^{s}$ & $-01^{\circ} 35^{\prime} 08^{\prime \prime}$ & $11^{\mathrm{m}} 40$ & 1.41 & 9.625 & $9^{\mathrm{m}} 101$ & 8.984 \\
\hline Check & TYC 5163-1652-1 & $20^{h} 23^{m} 09^{s}$ & $-01^{\circ} 42^{\prime} 16^{\prime \prime}$ & 11.70 & 1.06 & 9.924 & 9.424 & $9^{\mathrm{m}} \cdot 29$ \\
\hline Variable & $\mathrm{BD}+113024$ & $16^{h} 41^{m} 53^{s}$ & $+11^{\circ} 40^{\prime} 21^{\prime \prime}$ & $10^{\mathrm{m}} 13$ & $1 \mathrm{~m} \cdot 10$ & 7.890 & 7.294 & 7.077 \\
\hline Comparison & $\mathrm{BD}+113026 \mathrm{p}$ & $16^{h} 42^{m} 26^{s}$ & $+11^{\circ} 40^{\prime} 55^{\prime \prime}$ & 10.59 & 0.56 & 9.467 & 9.188 & $9^{m} .132$ \\
\hline Check & $\mathrm{BD}+113026$ & $16^{h} 42^{m} 28^{s}$ & $+11^{\circ} 41^{\prime} 35^{\prime \prime}$ & 9.90 & 0.44 & 8.953 & 8.773 & 8. 698 \\
\hline
\end{tabular}

ANOVA method. In the first step of the analysis, we determine photometric period of each observing season. Second, we phased the light curve of that season with its resulting photometric period. Finally, we fit a cubic spline to the phase-folded light curve, and determine minimum and maximum brightnesses. The mean brightness and the amplitude of the light curve are calculated from determined minimum and maximum brightnesses. We tabulate our analysis results in Table 5 We plot seasonal light curves of each target in the appendix.

For any of the target star, the number of sets listed in Table 5 is insufficient for evaluation of any correlation between parameters, e.g. the photometric period and the light curve amplitude, the amplitude and the brightness, or the photometric period and the mean brightness. For example, plotted photometric periods in Figure 3 do not seem to be correlated with the mean brightness. However, considerable discontinuities in the photometric data, and observing seasons where the photometric period could not be determined due to the very low light curve amplitude reduce the number of useful datasets for analysis, thus may cause misinterpretation of the real situation. Still, we find average photometric periods as $21.49,18.17,25^{\mathrm{d}} .61$ and 26.11 for $\mathrm{BD}+113024$, BD+13 5000, TYC 3557-919-1 and TYC 5163-1764-1, respectively. We use the distribution range of photometric periods and estimate $\Delta P / P=\left(P_{\max }-P_{\min }\right) / P_{e q}$, where $P_{\max }$ and $P_{\min }$ denote calculated maximum and minimum photometric periods for corresponding target, and $P_{e q}$ is equatorial rotation period, which is adopted as the mean value of the measured rotation periods for each target, 

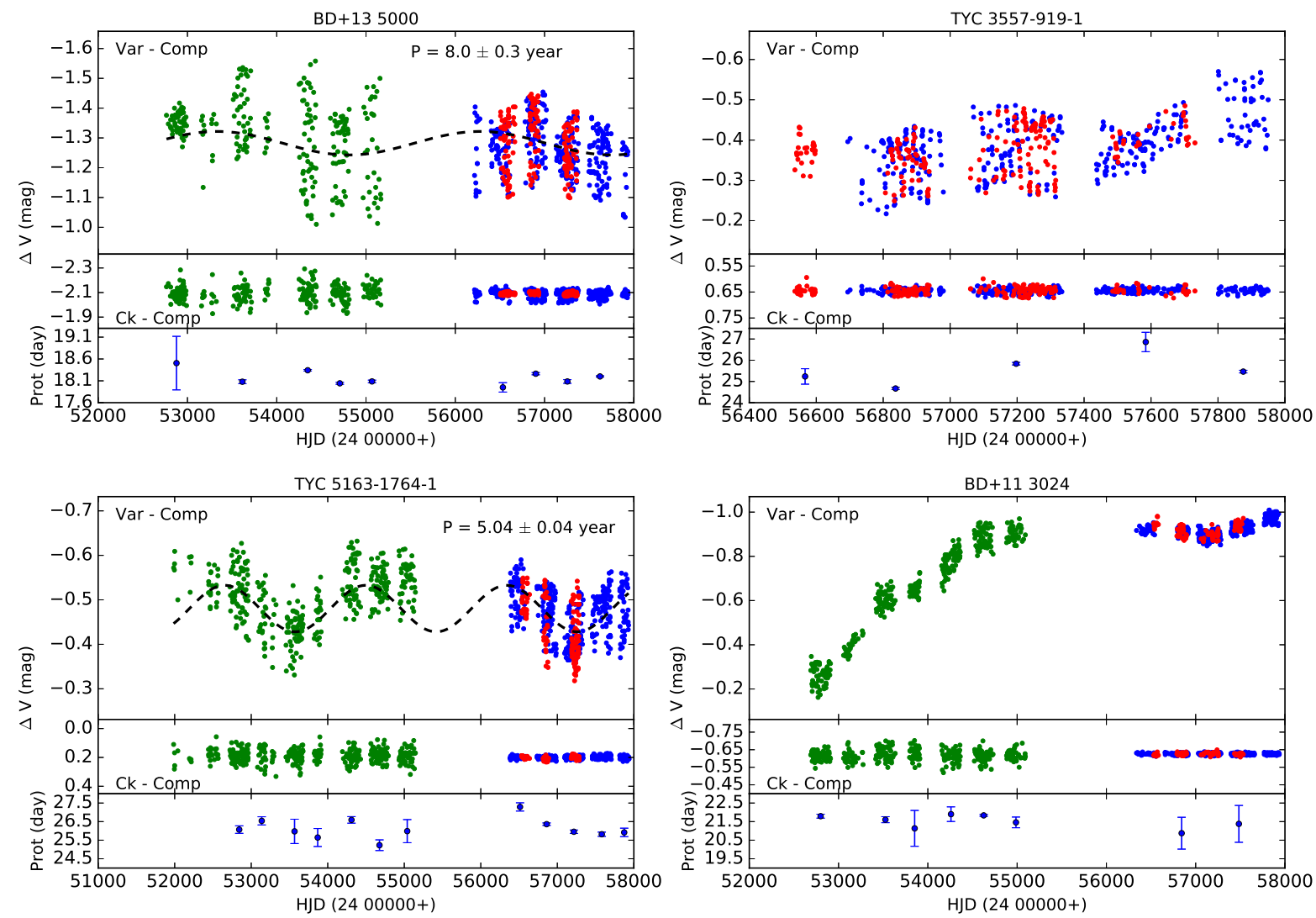

Fig. 3 Differential photometry of program stars. Var, comp and ck denote variable, comparison and check stars, respectively. Black dashed lines inside plot windows of BD+13 5000 and TYC 5163-1764-1 indicate estimated period for long term brightness variation. The estimated periods are shown in each window together with their uncertainties. Green, red and blue filled points show photometry from ASAS, T60, and ASAS-SN, respectively. Lowermost panel of each window shows seasonal photometric periods (see Section 3.3.2, and Table 5).

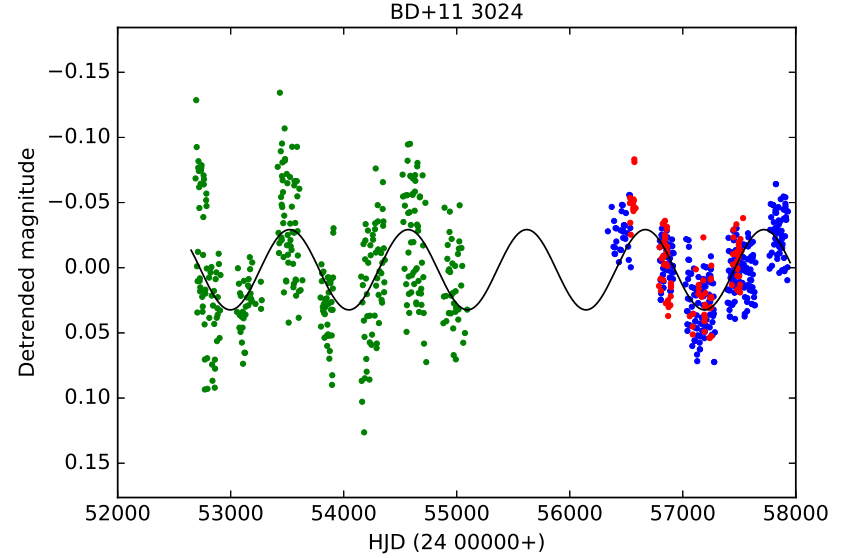

Fig. 4 2.87-yr cyclic brightness variation (black curve) detected in the de-trended photometric data of BD+113024. Meaning of colors are the same as Figure 3.

given in Table 5. Using periods listed in Table 5, we find $\Delta P / P$ values as $0.047,0.031,0.086,0.079$ for $\mathrm{BD}+113024$, BD+13 5000, TYC 3557-919-1 and TYC 5163-1764-1, respectively.

\section{Summary and discussion}

We present analysis of spectroscopic and long-term photometric observations of four cool stars, BD+113024, BD+13 5000, TYC 3557-919-1 and TYC 5163-1764-1. Strong emission in $\mathrm{H}_{\alpha}$ and $\mathrm{Ca}$ II $\mathrm{H} \& \mathrm{~K}$ lines, which exceeds the continuum in the case of BD+13 5000 and TYC 3557919-1, and remarkable changes in mean brightness and light curve amplitude clearly indicate chromospheric activity on these stars. Observed emissions appear to be rotationally modulated. Furthermore, non-stable radial velocities of the target stars indicate that they might possibly be a member of a binary system. However, binary nature of these stars and rotational modulation of the emission features need to be confirmed by further observations, which would cover at least a rotational cycle.

Analysis of seasonal light curves makes possible to trace seasonal photometric periods. Distribution range of photometric periods enables us to calculate $\Delta P / P$, which gives some hints on the lower limit of the differential rotation, and spot evolution in time, which could also alter the distribution of the photometric period in time. Growth and decay of spot regions at different latitudes and longitudes (i.e. temporal and spatial evolution of spots) may cause variation in 
Table 4 Brief information on data collected from ASAS, ASAS-SN databases and T60 telescope. $\mathrm{N}$ and $\sigma$ show number of data points and standard deviation calculated from check-minus-comparison measurements for corresponding star.

\begin{tabular}{|c|c|c|c|}
\hline \multirow[b]{2}{*}{ Star } & \multicolumn{3}{|c|}{ ASAS } \\
\hline & $\begin{array}{l}\text { HJD range } \\
(2400000+)\end{array}$ & $\mathrm{N}$ & $\begin{array}{c}\sigma \\
(\mathrm{mag}) \\
\end{array}$ \\
\hline $\mathrm{BD}+135000$ & $52765-55167$ & 252 & 0.066 \\
\hline TYC 3557-919-1 & - & - & - \\
\hline TYC 5163-1764-1 & $51985-55145$ & 369 & 0.045 \\
\hline \multirow[t]{2}{*}{$\mathrm{BD}+113024$} & $52688-55093$ & 357 & 0.033 \\
\hline & \multicolumn{3}{|c|}{ ASAS-SN } \\
\hline Star & $\begin{array}{l}\text { HJD range } \\
(2400000+)\end{array}$ & $\mathrm{N}$ & $\begin{array}{c}\sigma \\
(\mathrm{mag}) \\
\end{array}$ \\
\hline $\mathrm{BD}+135000$ & $56216-57932$ & 354 & 0.029 \\
\hline TYC 3557-919-1 & $56695-57950$ & 371 & 0.009 \\
\hline TYC 5163-1764-1 & $56389-57931$ & 387 & 0.009 \\
\hline \multirow[t]{2}{*}{$\mathrm{BD}+113024$} & $56337-57930$ & 373 & 0.005 \\
\hline & \multicolumn{3}{|c|}{ T60 } \\
\hline Star & $\begin{array}{l}\text { HJD range } \\
(2400000+)\end{array}$ & $\mathrm{N}$ & $\begin{array}{c}\sigma \\
(\mathrm{mag})\end{array}$ \\
\hline $\mathrm{BD}+135000$ & $56506-57365$ & 164 & 0.010 \\
\hline TYC 3557-919-1 & $56536-57731$ & 220 & 0.014 \\
\hline TYC 5163-1764-1 & $56533-57285$ & 140 & 0.012 \\
\hline $\mathrm{BD}+113024$ & $56532-57534$ & 107 & 0.007 \\
\hline
\end{tabular}

photometric period (see, e.g. Fekel et al. 2002). Less number of datasets in Table 5 do not allow one to arrive at any conclusive results, however, we notice that calculated $\Delta P / P$ values are larger than the values of spotted stars studied in Hall \& Busby (1990).

We find average rotational period of $\mathrm{BD}+113024$ as $21 \mathrm{~d} .49$. Considering the uncertainties of seasonal periods in the Table 5, it is in agreement with the period reported by Bernhard (2008). We observe more precise agreement in the case of BD+135000, where our average period is 18.17 and the period reported by Kiraga (2012) is 18.14 . The average photometric period of TYC 3557-919-1 found in this study is $25^{\mathrm{d}} .61$, while it was found as 25.08 by Bernhard (2008). In summary, we observe no discrepancies between average photometric periods found in this study and the literature values.

Below, we discus our further findings for each star, in a separate paragraph.

$B D+135000$. Our atmospheric analysis indicates K0IV spectral type for the star. Measured radial velocities are distributed in a range of $90 \mathrm{~km} \mathrm{~s}^{-1}$, indicating that the star could be a spectroscopic binary. Very strong emission, which exceeds the continuum, is observed in $\mathrm{H}_{\alpha}$ and $\mathrm{Ca}$ II $\mathrm{H} \& \mathrm{~K}$ lines. These features are accompanied with a cyclic variation in the mean brightness with a period of $8.0 \pm 0.3$ year, which repeats itself for two times in the time base of the current photometric data, and indicates an activity cycle. In most cases, light curve amplitudes are larger than 0.19. All these findings suggest a very strong and cyclic chromospheric activity.
TYC 3557-919-1. Analysis of TFOSC spectra indicates KOIII-IV spectral type. Radial velocities appear to be variable, where the maximum difference among the measurements is to be $52 \mathrm{~km} \mathrm{~s}^{-1}$, suggesting the possibility of being spectroscopic binary. Similar to BD+13 5000, $\mathrm{H}_{\alpha}$ and $\mathrm{Ca}$ II $\mathrm{H} \& \mathrm{~K}$ lines exhibit very strong emission that exceeds the continuum. Available photometric data cover four years and only indicate a global brightening trend of $\sim 0 \mathrm{~m} 1$, with a seasonal light curve amplitude usually between 0.1 and $0 \mathrm{~m} 2$. These are clear evidence of very strong chromospheric activity.

TYC 5163-1764-1. Spectral type of the star is found as K2III. As in the cases of BD+13 5000 and TYC 3557-9191 , we observe unstable radial velocities in a range of 49 $\mathrm{km} \mathrm{s}^{-1}$, indicating possibility of being spectroscopic binary. Emission observed in $\mathrm{H}_{\alpha}$ and $\mathrm{Ca}$ II $\mathrm{H} \& \mathrm{~K}$ lines are weaker compared to BD+135000 and TYC 3557-919-1, but still clearly observed, reaching to the continuum level around $\mathrm{Ca}$ II $\mathrm{K}$ line. We find cyclic mean brightness variation with a period of $5.04 \pm 0.04$ year, repeating itself more than three times in the time span of the photometric data. Observed light curve amplitudes are between 0.075 and 0.154 . These findings suggest strong and cyclic chromospheric activity.

$B D+113024$. TFOSC spectra of this star indicate KOIIIIV spectral type. Measured radial velocities are distributed between smaller range $\left(17 \mathrm{~km} \mathrm{~s}^{-1}\right)$ compared to the other target stars, weakly indicates possibility of being spectroscopic binary. Strengths of $\mathrm{H}_{\alpha}$ and $\mathrm{Ca}$ II $\mathrm{H} \& \mathrm{~K}$ emissions of the star are very similar to the observed emissions in TYC 5163-1764-1. Photometric data of the star exhibit very dramatic brightness increase $(\sim 0.7)$ accompanied with a $2.87 \pm 0.12$ year cyclic brightness variation with 0.03 amplitude. The star might have multiple cycles if the global brightness increase is part of a longer term cyclic brightness change with a larger amplitude. This would not be surprising since multiple cycles appear to be observed often in active stars (Oláh et al. 2009). Seasonal light curve amplitude, usually below $0{ }^{\mathrm{m}} 1$, and interesting brightness variations make this star an attractive target for further studies.

Spectroscopic and photometric characteristics of BD+13 5000, TYC 3557-919-1 and TYC 5163-1764-1 are similar to the typical RS CVn stars with strong emission in their $\mathrm{Ca}$ II $\mathrm{H} \& \mathrm{~K}$ lines, cyclic mean brightness variation and remarkable light curve amplitude, e.g. V1149 Ori (Fekel \& Henry 2005, Jetsu et al. 2017), HD 208472 (Özdarcan et al. 2016, 2010), FG UMa (Fekel et al. 2002, Özdarcan et al. 2012), and many other targets studied in Oláh et al. (2009) and Jetsu et al. (2017). Individually, photometric behaviour of BD+113024 resembles RS CVn binary IS Vir (Fekel et al. 2002; Oláh et al. 2013), which possesses similar light curve amplitude, and a long term variation with an estimated period of 5-6 years (Oláh et al. 2013). Dramatic changes in the mean brightness of BD+113024 suggests strong spot activity. In this case, low light curve amplitude could be explained by either assuming that the distribution of spots is usually homogeneous on the surface 
Table 5 Brightness levels and photometric periods found from application of the ANOVA method to the seasonal light curves. In the first four columns, set number, beginning, end and mean heliocentric Julian dates of the corresponding light curve are given. Photometric period and its uncertainty $(P$ and $\sigma(P))$ are in unit of day. The maximum, minimum and mean brightnesses, and the light curve amplitude $(A)$ are given in magnitudes. In the last column, $N$ shows number of data points used in the analysis.

\begin{tabular}{|c|c|c|c|c|c|c|c|c|c|c|}
\hline \multicolumn{11}{|c|}{$\mathrm{BD}+113024$} \\
\hline Set & $\begin{array}{c}\text { HJD min. } \\
(2400000+)\end{array}$ & $\begin{array}{c}\text { HJD max. } \\
(2400000+)\end{array}$ & $\begin{array}{c}\text { HJD mean } \\
(2400000+)\end{array}$ & $P$ & $\sigma(P)$ & $\max$ & $\min$ & mean & $A$ & $N$ \\
\hline 1 & 52688.8799 & 52909.4910 & 52799.1855 & 21.79 & 0.11 & -0.323 & -0.206 & -0.265 & 0.117 & 65 \\
\hline 2 & 53415.8809 & 53634.4802 & 53525.1806 & 21.60 & 0.16 & -0.650 & -0.553 & -0.602 & 0.097 & 62 \\
\hline 3 & 53794.8888 & 53909.7268 & 53852.3078 & 21.14 & 0.97 & -0.677 & -0.622 & -0.649 & 0.055 & 39 \\
\hline 4 & 54154.8879 & 54363.4964 & 54259.1922 & 21.90 & 0.39 & -0.825 & -0.731 & -0.778 & 0.094 & 57 \\
\hline 5 & 54520.8874 & 54730.4803 & 54625.6839 & 21.84 & 0.05 & -0.938 & -0.831 & -0.884 & 0.107 & 60 \\
\hline 6 & 54881.8918 & 55092.4985 & 54987.1952 & 21.46 & 0.29 & -0.952 & -0.874 & -0.913 & 0.078 & 38 \\
\hline 7 & 56789.5162 & 56896.2329 & 56842.8746 & 20.88 & 0.86 & -0.926 & -0.875 & -0.901 & 0.051 & 37 \\
\hline 8 & 57434.6040 & 57533.3902 & 57483.9971 & 21.38 & 0.99 & -0.964 & -0.923 & -0.943 & 0.041 & 27 \\
\hline \multicolumn{11}{|c|}{$\mathrm{BD}+135000$} \\
\hline Set & $\begin{array}{c}\text { HJD min. } \\
(2400000+)\end{array}$ & $\begin{array}{l}\text { HJD max. } \\
(2400000+)\end{array}$ & $\begin{array}{l}\text { HJD mean } \\
(2400000+)\end{array}$ & $P$ & $\sigma(P)$ & $\max$ & $\min$ & mean & $A$ & $N$ \\
\hline 1 & 52764.9261 & 52989.5301 & 52877.2281 & 18.51 & 0.61 & -1.378 & -1.320 & -1.349 & 0.058 & 52 \\
\hline 2 & 53512.9205 & 53717.5263 & 53615.2234 & 18.08 & 0.04 & -1.507 & -1.263 & -1.385 & 0.244 & 45 \\
\hline 3 & 54250.9308 & 54443.5358 & 54347.2333 & 18.34 & 0.02 & -1.506 & -1.062 & -1.284 & 0.444 & 52 \\
\hline 4 & 54601.9120 & 54811.5315 & 54706.7218 & 18.04 & 0.02 & -1.360 & -1.047 & -1.203 & 0.313 & 50 \\
\hline 5 & 54967.9262 & 55166.5666 & 55067.2464 & 18.09 & 0.03 & -1.451 & -1.063 & -1.257 & 0.388 & 31 \\
\hline 6 & 56397.1295 & 56669.1541 & 56533.1418 & 17.95 & 0.11 & -1.352 & -1.160 & -1.256 & 0.192 & 110 \\
\hline 7 & 56782.0933 & 57020.6935 & 56901.3934 & 18.26 & 0.03 & -1.424 & -1.171 & -1.298 & 0.253 & 132 \\
\hline 8 & 57132.1243 & 57378.7314 & 57255.4279 & 18.09 & 0.04 & -1.349 & -1.159 & -1.254 & 0.190 & 161 \\
\hline 9 & 57496.1318 & 57745.6977 & 57620.9148 & 18.20 & 0.02 & -1.337 & -1.094 & -1.215 & 0.243 & 83 \\
\hline \multicolumn{11}{|c|}{ TYC 3557-919-1 } \\
\hline Set & $\begin{array}{c}\text { HJD min. } \\
(2400000+)\end{array}$ & $\begin{array}{c}\text { HJD max. } \\
(2400000+)\end{array}$ & $\begin{array}{l}\text { HJD mean } \\
(2400000+)\end{array}$ & $P$ & $\sigma(P)$ & $\max$ & $\min$ & mean & $A$ & $N$ \\
\hline 1 & 56536.2497 & 56597.1793 & 56566.7145 & 25.24 & 0.36 & -0.431 & -0.292 & -0.361 & 0.139 & 29 \\
\hline 2 & 56694.1694 & 56979.6930 & 56836.9312 & 24.67 & 0.05 & -0.412 & -0.251 & -0.332 & 0.161 & 179 \\
\hline 3 & 57060.6618 & 57333.7025 & 57197.1822 & 25.84 & 0.06 & -0.462 & -0.283 & -0.373 & 0.179 & 203 \\
\hline 4 & 57437.1658 & 57731.1923 & 57584.1791 & 26.86 & 0.46 & -0.432 & -0.363 & -0.398 & 0.069 & 132 \\
\hline 5 & 57801.1689 & 57949.8032 & 57875.4860 & 25.46 & 0.06 & -0.567 & -0.398 & -0.483 & 0.169 & 48 \\
\hline \multicolumn{11}{|c|}{ TYC 5163-1764-1 } \\
\hline Set & $\begin{array}{c}\text { HJD min. } \\
(2400000+)\end{array}$ & $\begin{array}{c}\text { HJD max. } \\
(2400000+)\end{array}$ & $\begin{array}{l}\text { HJD mean } \\
(2400000+)\end{array}$ & $P$ & $\sigma(P)$ & $\max$ & $\min$ & mean & $A$ & $N$ \\
\hline 1 & 52721.8999 & 52964.5131 & 52843.2065 & 26.07 & 0.20 & -0.594 & -0.463 & -0.528 & 0.131 & 66 \\
\hline 2 & 53082.9093 & 53191.7672 & 53137.3383 & 26.54 & 0.22 & -0.541 & -0.392 & -0.467 & 0.149 & 25 \\
\hline 3 & 53454.9023 & 53677.5511 & 53566.2267 & 25.98 & 0.65 & -0.457 & -0.382 & -0.420 & 0.075 & 61 \\
\hline 4 & 53823.8992 & 53913.7795 & 53868.8394 & 25.64 & 0.48 & -0.535 & -0.407 & -0.471 & 0.128 & 22 \\
\hline 5 & 54191.8947 & 54428.5135 & 54310.2041 & 26.59 & 0.18 & -0.604 & -0.488 & -0.546 & 0.116 & 53 \\
\hline 6 & 54559.9012 & 54792.5118 & 54676.2065 & 25.23 & 0.29 & -0.574 & -0.494 & -0.534 & 0.080 & 63 \\
\hline 7 & 54931.8943 & 55145.5269 & 55038.7106 & 25.99 & 0.62 & -0.593 & -0.500 & -0.546 & 0.093 & 42 \\
\hline 8 & 56389.0940 & 56636.1713 & 56512.6327 & 27.29 & 0.22 & -0.553 & -0.461 & -0.507 & 0.092 & 102 \\
\hline 9 & 56735.1287 & 56984.6854 & 56859.9071 & 26.36 & 0.07 & -0.526 & -0.376 & -0.451 & 0.150 & 118 \\
\hline 10 & 57094.9021 & 57337.5197 & 57216.2109 & 25.95 & 0.09 & -0.501 & -0.364 & -0.433 & 0.137 & 170 \\
\hline 11 & 57457.1418 & 57705.5100 & 57581.3259 & 25.82 & 0.11 & -0.525 & -0.406 & -0.465 & 0.119 & 91 \\
\hline 12 & 57817.1547 & 57930.9784 & 57874.0666 & 25.92 & 0.23 & -0.554 & -0.400 & -0.477 & 0.154 & 46 \\
\hline
\end{tabular}


of the star, or assuming the inclination of the rotational axis is low, as in the case of IS Vir, thus causing low light curve amplitude.

High resolution optical spectroscopy is required to determine more precise atmospheric properties and projected rotational velocities, as well as to trace radial velocities in order to check whether each of these stars is single or a member of a binary system. Determination of projected rotational velocities from high resolution spectroscopy would help to restrict the stellar radius, which is one of the key parameter to calculate stellar luminosity, i.e. vertical position of a star in HR diagram. Further time series photometric observations would enable one to trace activity cycles and study the relation between cycle length and rotation period (e.g. Oláh \& Strassmeier 2002), as well as surface differential rotation via distribution of seasonal photometric periods in time (Hall \& Busby 1990, Özdarcan et al. 2012, 2010).

Acknowledgements. We thank to TÜBITAK for a partial support in using RTT150 (Russian-Turkish 1.5-m telescope in Antalya) with project number 14BRTT150-678, and T60 telescope with project number 13CT60-504. We also thank our referee, Dr. Katalin Oláh, for her useful comments that improve the paper. This research has made use of the SIMBAD database, operated at CDS, Strasbourg, France.

\section{References}

Bernhard, K. 2008, Open European Journal on Variable Stars, 78, 1

Blanco-Cuaresma, S., Soubiran, C., Heiter, U., \& Jofré, P. 2014, A\&A, 569, A111

Boyle, B. J., Wilkes, B. J., \& Elvis, M. 1997, MNRAS, 285, 511

Cutri, R. M., Skrutskie, M. F., van Dyk, S., et al. 2003, VizieR Online Data Catalog, 2246

Fekel, F. C. \& Henry, G. W. 2005, AJ, 129, 1669

Fekel, F. C., Henry, G. W., Eaton, J. A., Sperauskas, J., \& Hall, D. S. 2002, AJ, 124, 1064

Fitzpatrick, M. J. 1993, in Astronomical Society of the Pacific Conference Series, Vol. 52, Astronomical Data Analysis Software and Systems II, ed. R. J. Hanisch, R. J. V. Brissenden, \& J. Barnes, 472

Gray, R. O. \& Corbally, C. J. 1994, AJ, 107, 742

Gustafsson, B., Edvardsson, B., Eriksson, K., et al. 2008, A\&A, 486, 951

Haakonsen, C. B. \& Rutledge, R. E. 2009, ApJS, 184, 138

Hall, D. S. \& Busby, M. R. 1990, in NATO Advanced Science Institutes (ASI) Series C, Vol. 319, NATO Advanced Science Institutes (ASI) Series C, 377

Henry, G. W. \& Eaton, J. A., eds. 1995, Astronomical Society of the Pacific Conference Series, Vol. 79, Robotic telescopes : current capabilities, present developments, and future prospects for automated astronomy : proceedings of a symposium held as part of the 106th annual meeting of the Astronomical Society of the Pacific, Flagstaff, Arizona, 28-30 June 1994

Hoffman, D. I., Harrison, T. E., \& McNamara, B. J. 2009, AJ, 138, 466

Høg, E., Fabricius, C., Makarov, V. V., et al. 2000, A\&A, 355, L27

Jetsu, L., Henry, G. W., \& Lehtinen, J. 2017, ApJ, 838, 122

Kiraga, M. 2012, Acta Astron., 62, 67
Kochanek, C. S., Shappee, B. J., Stanek, K. Z., et al. 2017, PASP, 129, 104502

Lloyd, C., Schirmer, J., Bernhard, K., \& Frank, P. 2011, Open European Journal on Variable Stars, 136, 1

Lomb, N. R. 1976, Ap\&SS, 39, 447

Oláh, K., Kolláth, Z., Granzer, T., et al. 2009, A\&A, 501, 703

Oláh, K., Moór, A., Strassmeier, K. G., Borkovits, T., \& Granzer, T. 2013, Astronomische Nachrichten, 334, 625

Oláh, K. \& Strassmeier, K. G. 2002, Astronomische Nachrichten, 323,361

Özdarcan, O., Carroll, T. A., Künstler, A., et al. 2016, A\&A, 593, A123

Özdarcan, O., Evren, S., \& Henry, G. W. 2012, Astronomische Nachrichten, 333, 138

Özdarcan, O., Evren, S., Strassmeier, K. G., Granzer, T., \& Henry, G. W. 2010, Astronomische Nachrichten, 331, 794

Pojmanski, G. 1997, Acta Astron., 47, 467

Pojmanski, G. 2002, Acta Astron., 52, 397

Pojmanski, G., Pilecki, B., \& Szczygiel, D. 2005, Acta Astron., 55,275

Roettenbacher, R. M., Harmon, R. O., Vutisalchavakul, N., \& Henry, G. W. 2011, AJ, 141, 138

Ryabchikova, T., Piskunov, N., Kurucz, R. L., et al. 2015, Phys. Scr, 90, 054005

Scargle, J. D. 1982, ApJ, 263, 835

Schwarzenberg-Czerny, A. 1996, ApJ, 460, L107

Shappee, B. J., Prieto, J. L., Grupe, D., et al. 2014, ApJ, 788, 48

Sharma, K., Prugniel, P., \& Singh, H. P. 2016, A\&A, 585, A64

Stellingwerf, R. F. 1978, ApJ, 224, 953

Strassmeier, K. G., Boyd, L. J., Epand, D. H., \& Granzer, T. 1997 , PASP, 109, 697

Tonry, J. \& Davis, M. 1979, AJ, 84, 1511

Voges, W., Aschenbach, B., Boller, T., et al. 1999, A\&A, 349, 389

Woźniak, P. R., Vestrand, W. T., Akerlof, C. W., et al. 2004, AJ, 127,2436

Zickgraf, F.-J., Engels, D., Hagen, H.-J., Reimers, D., \& Voges, W. 2003, A\&A, 406, 535

\section{A Seasonal light curves and phase folded radial velocities}



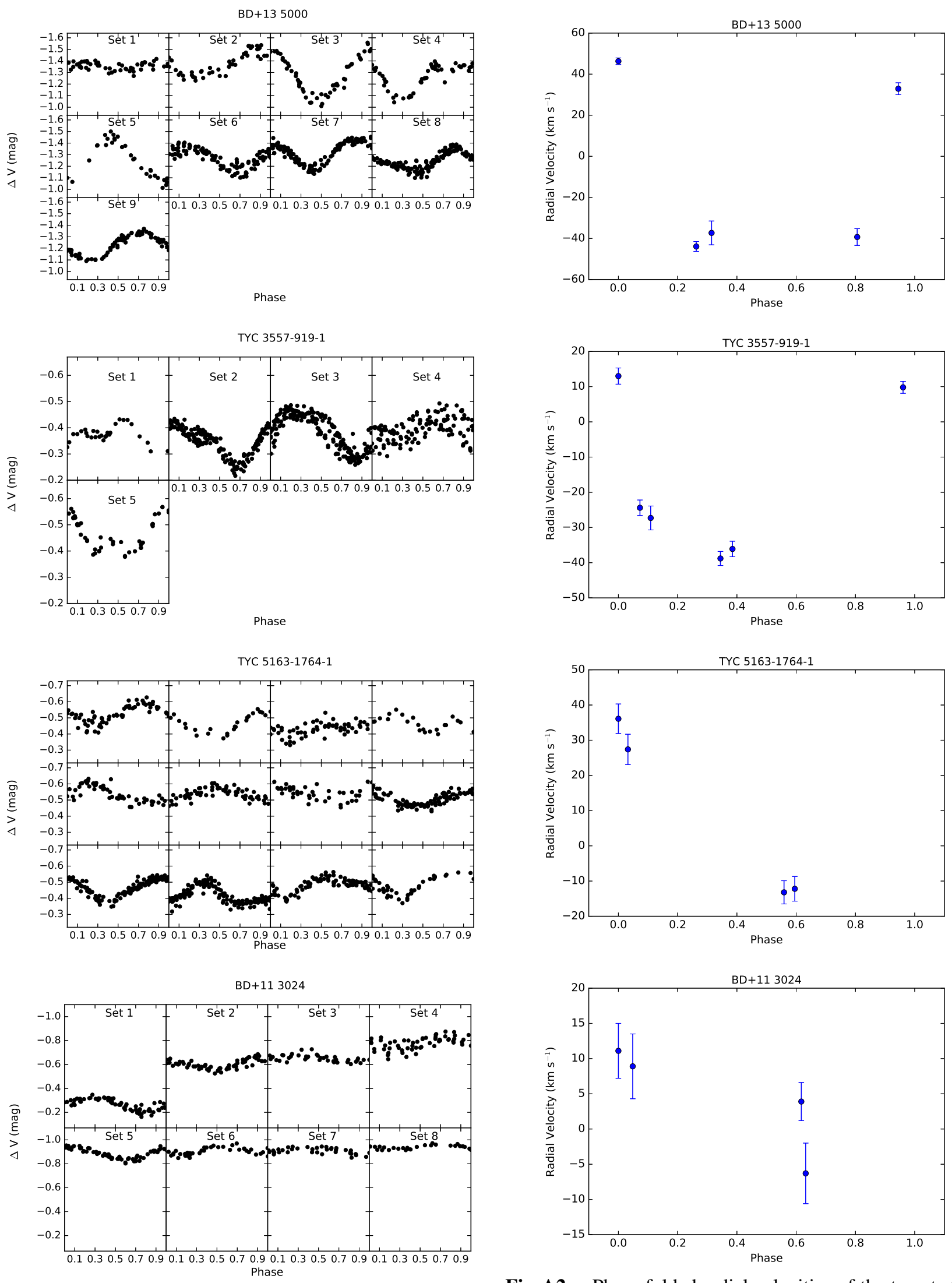

Fig. A1 Phase folded seasonal light curves of the target stars. Phase folding is done with the calculated photometric period listed in Table 5 and heliocentric Julian date of the initial data point of corresponding light curve.

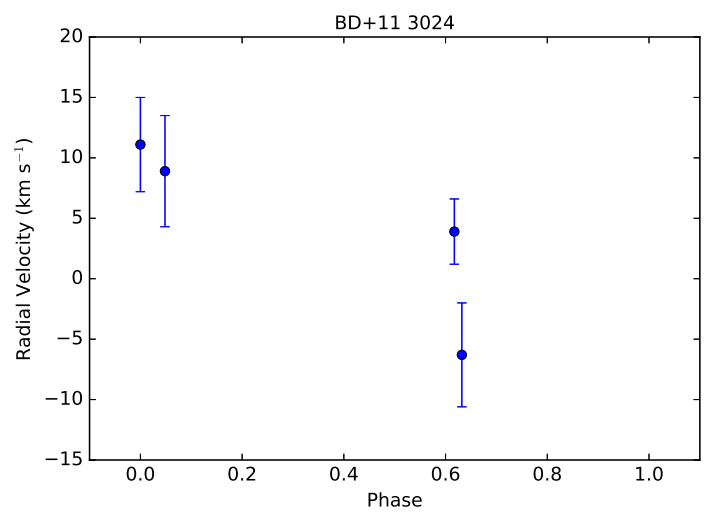

Fig. A2 Phase folded radial velocities of the target stars. Phase folding is done with respect to the time of the observed positive maximum velocity (ephemeris, see Table 1 ) and calculated mean photometric period read from Table 5 